\title{
Metallic nanograins: Spatially nonuniform pairing induced by quantum confinement
}

\author{
M. D. Croitoru, ${ }^{1,2, *}$ A. A. Shanenko, ${ }^{3}$ C. C. Kaun, ${ }^{2}$ and F. M. Peeters ${ }^{3}$ \\ ${ }^{1}$ Institut für Theoretische Physik III, Universität Bayreuth, DE-95440 Bayreuth, Germany \\ ${ }^{2}$ Research Center for Applied Sciences, Academia Sinica, 11529 Taipei, Taiwan \\ ${ }^{3}$ Departement Fysica, Universiteit Antwerpen, Groenenborgerlaan 171, BE-2020 Antwerpen, Belgium \\ (Received 13 July 2010; revised manuscript received 29 April 2011; published 6 June 2011)
}

\begin{abstract}
It is well known that the formation of discrete electron levels strongly influences the pairing in metallic nanograins. Here, we focus on another effect of quantum confinement in superconducting grains that was not studied previously, i.e., spatially nonuniform pairing. This effect is very significant when single-electron levels form bunches and/or a kind of shell structure. We find that, in highly symmetric grains, the order parameter can exhibit variations with position by an order of magnitude. Nonuniform pairing is closely related to a quantumconfinement-induced modification of the pairing-interaction matrix elements and size-dependent pinning of the chemical potential to groups of degenerate or nearly degenerate levels. For illustrative purposes, we consider spherical metallic nanograins and also rectangular shapes. We show that the relevant matrix elements are, as a rule, enhanced in the presence of quantum confinement, which favors spatial variations of the order parameter, compensating the corresponding energy cost. The size-dependent pinning of the chemical potential further increases the spatial variation of the pair condensate. The role of nonuniform pairing is smaller in less symmetric confining geometries and/or in the presence of disorder. However, it always remains of importance when the energy spacing between discrete electron levels $\delta$ is approaching the scale of the bulk gap $\Delta_{B}$, i.e., $\delta>0.1-0.2 \Delta_{B}$.

DOI: 10.1103/PhysRevB.83.214509

PACS number(s): 74.20.Fg, 74.78.Na
\end{abstract}

\section{INTRODUCTION}

Quantum confinement plays a fundamental role in superconductors with nanoscale dimensions. Interplay of quantum confinement and pairing correlations results in important qualitative changes in the superconductor characteristics. ${ }^{1-17}$ Because of technological reasons, quasi-zero-dimensional superconducting structures (i.e., ensembles of small grains) were the first where this interplay was investigated experimentally. Initial attempts by Giaever and Zeller at the end of the 1960s used tunneling studies on large ensembles of superconducting particles. ${ }^{19}$ Since that time, most of the studies on superconducting correlations in grains were performed with grain powders ${ }^{20,21}$ or on films made of crystalline granules separated by amorphous intergranular space. ${ }^{22,23}$ In the pioneering work of Ralph et al., ${ }^{24,25}$ the discrete electron spectrum was measured for a single grain. Their technique (single-electron tunneling spectroscopy) enabled them to probe superconducting correlations in an individual Al grain. Very recently, Scanning tunneling microscope(STM) was used to detect the superconducting gap of an isolated ultrasmall lead grain deposited onto a silicon substrate (see, e.g., Refs. 26 and 27). These advances opened new prospects to examine superconductivity in individual metallic nanograins with unprecedented detail, e.g., to investigate how quantum confinement can influence the superconducting correlations.

The main feature of a superconducting nanograin that makes it different from a bulk superconductor is the formation of discrete electron levels with average energy spacing $\delta \approx$ $2 \pi^{2} \hbar^{2} /\left(m k_{F} V\right)$, with $k_{F}$ the bulk Fermi wave number and $V$ the system volume. It can be of the same order as the bulk gap $\Delta_{B}$, or even larger in the case of ultrasmall nanograins. Therefore, size quantization of the electron spectrum can have a substantial impact on the basic superconducting characteristics of such quasi-zero-dimensional superconducting systems.
The understanding of the fundamental properties of superconducting correlations in low-dimensional structures, in particular in isolated metallic grains, has experienced a remarkable development in the last two decades. Theoretical aspects, which have attracted the most attention, are as follows. The problem of the breakdown of BCS superconductivity in ultrasmall metallic grains was addressed in several papers. ${ }^{4,28,29}$ Finite-size enhancement of pairing in mesoscopic grains was considered (see Ref. 30 and references therein). Effects of the shell structure in the single-electron spectrum on superconducting correlations were pointed out for nanograins ${ }^{8,17}$ and ultrasmall metallic clusters. ${ }^{10,11}$ The ground-state properties of the BCS pairing Hamiltonian of ultrasmall grains were considered beyond the mean-field approximation using the Richardson exact solution $^{7-9}$ and the single shell model. ${ }^{18}$

Nonuniform spatial distribution of the superconducting condensate was not investigated in these and other papers, and a spatially uniform pairing was, as a rule, assumed. However, the translational invariance is broken in nanograins due to quantum confinement, which leads to a position-dependent order parameter. So, the question arises as to when the breakdown of the translational symmetry in metallic superconducting nanograins will significantly influence the superconducting correlations. We note that the position dependence of the order parameter is directly related to the following two points, specific of nanograins. First, single-electron levels can form bunches and even a kind of shell structure (in symmetric confining geometries). In this case, the chemical potential $\mu$ can be pinned to groups of condensed levels (i.e., nearly degenerate or degenerate levels). This is of importance because the density of states in the vicinity of $\mu$ strongly influences the superconducting correlations. In other words, such a pinning plays the role of a filter that selects the contribution of a particular single-electron shell (or of a group of close levels) to the superconducting order parameter that is, as 
a rule, spatially nonuniform. Second, a position-dependent order parameter means that the pairing gaps become strongly dependent on the relevant quantum numbers, similar to the case of superfluid fermions in atomic traps and nuclei (see, e.g., Ref. 31). This is connected with a confinement-induced modification (as compared to $-g / V$, with $g>0$ the coupling constant and $V$ the volume) of the matrix elements controlling the scattering of the time-reversed states: such modifications strongly depend on the relevant quantum numbers. To our knowledge, at present, there is only one $\operatorname{paper}^{17}$ (see also the following up extended publication of the same authors, i.e., Ref. 32) where the energy dependence of the matrix elements for superconducting metallic grains was considered for the particular case of chaotic grains. Such a dependence is related to a nonuniform spatial distribution of the pair condensate. However, any systematic analysis of nonuniform pairing was beyond the scope of those papers.

Thus, the aim of this paper is to investigate spatially nonuniform pairing in metallic nanograins, which was not studied in previous publications. For illustrative purposes, we consider metallic spherical nanograins, where the spatial dependence of the superconducting condensate is pronounced (the order parameter can vary with position by an order of magnitude). In less symmetric confining geometries (here we employ the rectangular shape) and/or in the presence of disorder, spatial variations of the order parameter are reduced. However, our study confirms that nonuniform pairing is always of importance when the interlevel spacing $\delta$ is of the order of the bulk gap $\Delta_{B}$. Any remaining grouping of single-electron levels, which is always present in real samples, even strengthens the effect of interest. We work in the mean-field approximation and, thus, stay in the regime $\delta \lesssim \Delta_{B}$. Below, we consider Sn and Al with $\Delta_{B}=0.616$ and $0.25 \mathrm{meV}$, respectively (for the parameters used below). By using the above values of $\Delta_{B}$, we find that the mean-field approach is valid for $D>6-8 \mathrm{~nm}$, with $D$ the sphere diameter.

Our paper is organized as follows. In Sec. II, we outline the formalism as to how to obtain a self-consistent solution to the problem. In Sec. III, we present our numerical results. In particular, in Sec. III A, we investigate the effects of quantum confinement on pairing correlations through the modifications of the matrix elements of the pairing interaction and the size-dependent pinning of $\mu$ to single-electron shells. Section III B is focused on a spatial distribution of the pair condensate and its relation to modifications of the matrix elements and the size-dependent pinning of $\mu$. In Sec. III C, we discuss the interplay of Andreev reflection with quantum confinement that results in the formation of Andreev-type states and significant dependence of the pairing gaps on the relevant quantum numbers. A short summary and discussions are given in Sec. IV, where our consideration of spherical grains is also supplemented by results for rectangular-shape nanoparticles, where the shell structure is dissolved and the single-electron spectrum becomes almost equidistant.

\section{FORMALISM}

The reduction of the system to the nanometer scale leads to the formation of a discrete electron spectrum. Moreover, in the presence of quantum confinement, the translational invariance of the system is broken, and the superconducting order parameter is position dependent, i.e., $\Delta=\Delta(\mathbf{r})$. For the mean-field treatment of such a situation, it is appropriate to use the Bogoliubov-de Gennes (BdG) equations, ${ }^{33,34}$ which can be written as

$$
\begin{gathered}
E_{i}\left|u_{i}\right\rangle=\widehat{H}_{e}\left|u_{i}\right\rangle+\widehat{\Delta}\left|v_{i}\right\rangle, \\
E_{i}\left|v_{i}\right\rangle=\widehat{\Delta}^{*}\left|u_{i}\right\rangle-\widehat{H}_{e}^{*}\left|v_{i}\right\rangle,
\end{gathered}
$$

where $E_{i}$ stands for the Bogoliubov-quasiparticle (bogolon) energy $\widehat{\Delta}=\Delta(\widehat{\mathbf{r}})$ (with $\widehat{\mathbf{r}}$ the position operator) and the singleelectron Hamiltonian is referred to the chemical potential $\mu$, i.e.,

$$
\widehat{H}_{e}(\mathbf{r})=\frac{\widehat{\mathbf{p}}^{2}}{2 m_{e}}+V(\widehat{\mathbf{r}})-\mu .
$$

We remark that any magnetic effects are beyond the scope of this paper. For simplicity, the confining interaction $V(\mathbf{r})$ is taken as zero inside the specimen and infinite outside: $V(\mathbf{r})=V_{B} \vartheta(R-\rho)$ with the barrier potential $V_{B} \rightarrow \infty$ ( $R=D / 2$ and $\rho$ is the radial coordinate for the spherical confining geometry).

As a mean-field approach, the BdG equations should be solved in a self-consistent manner:

$$
\Delta(\mathbf{r})=g \sum_{i}\left\langle\mathbf{r} \mid u_{i}\right\rangle\left\langle v_{i} \mid \mathbf{r}\right\rangle \tanh \left(\frac{\beta E_{i}}{2}\right),
$$

where $g>0$ is the coupling constant for the effective electronelectron interaction approximated by the delta-function potential, i.e., $\left\langle\mathbf{r}, \mathbf{r}^{\prime}|\Phi| \mathbf{r}, \mathbf{r}^{\prime}\right\rangle=-g \delta\left(\mathbf{r}-\mathbf{r}^{\prime}\right)$. The sum in Eq. (3) runs over the states with the single-electron energy

$$
\xi_{i}=\left[\left\langle u_{i}\left|\widehat{H}_{e}\right| u_{i}\right\rangle+\left\langle v_{i}\left|\widehat{H}_{e}\right| v_{i}\right\rangle\right] \in\left[-\hbar \omega_{D}, \hbar \omega_{D}\right],
$$

with $\omega_{D}$ the Debye frequency. As is known, the solution of the BdG equations has two branches: $(i,+)$ and $(i,-)$ (see Ref. 35) for which we have $E_{i,+}>0$ and $E_{i,-}<0$. The sum in Eq. (3) should be taken over the physical states [the $(i,+)$ branch], i.e., $E_{i}=E_{i,+}$.

For a given mean electron density $n_{e}$, the chemical potential $\mu$ is determined from

$$
n_{e}=\frac{2}{V} \sum_{i}\left[f_{i}\left\langle u_{i} \mid u_{i}\right\rangle+\left(1-f_{i}\right)\left\langle v_{i} \mid v_{i}\right\rangle\right],
$$

with $V=\frac{4}{3} \pi R^{3}$ the volume of the spherical grain. For conventional superconductors, the energy gap is typically much smaller than the chemical potential. As a result, $\mu$ stays nearly the same when passing from the normal state to the superconducting one. ${ }^{34}$ Therefore, one can solve Eq. (5) in the absence of superconducting order $[\Delta(\mathbf{r})=0]$.

In a spherical nanograin, because of symmetry reasons, the order parameter depends only on the radial coordinate, i.e., $\Delta=\Delta(\rho)$. Therefore, the pseudospinor in the particlehole space can be characterized by the quantum numbers of the angular momentum, i.e., $(l, m)$. The angular part of the pseudospinor $\Psi_{i}$ is given by the spherical harmonics $Y_{l m}(\theta, \varphi)$ in polar coordinates $(\rho, \theta, \varphi)$, i.e.,

$$
\left\langle\mathbf{r} \mid \Psi_{i}\right\rangle=Y_{l m}(\theta, \varphi)\left(\begin{array}{l}
u_{j l}(\rho) \\
v_{j l}(\rho)
\end{array}\right),
$$


where $i=\{j, l, m\}$, with $j$ the radial quantum number associated with the quantum-confinement boundary conditions

$$
\left.u_{j l}(\rho)\right|_{\rho=R}=\left.v_{j l}(\rho)\right|_{\rho=R}=0 .
$$

To solve the BdG equations (1a) and (1b) numerically, $u_{j l}(\rho)$ and $v_{j l}(\rho)$ are expanded in the eigenfunctions of the single-electron Hamiltonian $\widehat{H}_{e}$ [see Eq. (2)]. In addition, iterations should be invoked to account for the self-consistency relation given by Eq. (3). This program is significantly simplified by keeping only the pairing of the time-reversed states, ${ }^{36}$ which is a standard approximation for the problem of superconducting correlations in nanograins. We note that such an approximation is fully justified for small enough grains, where the interlevel spacing $\delta$ is approaching the scale of the order of the bulk gap $\Delta_{B}$. For larger grains, when $\delta \ll \Delta_{B}$, some corrections can be expected. However, our numerical study of the BdG equations shows that such corrections do not exceed several percent for $\delta \sim 0.1 \Delta_{B}$. In the framework of the $\mathrm{BdG}$ equations, the pairing of the time-reversed states is realized through the so-called Anderson's approximate solution for which the particlelike and holelike wave functions are assumed to be proportional to the single-electron wave function. It means that

$$
u_{j l}(\rho)=\mathcal{U}_{j l} \chi_{j l}(\rho), u_{j l}(\rho)=\mathcal{V}_{j l} \chi_{j l}(\rho),
$$

with the radial part of the single-electron wave function given by

$$
\chi_{j l}(\rho)=\frac{\sqrt{2}}{R^{3 / 2} j_{l+1}\left(\alpha_{j l}\right)} j_{l}\left(\alpha_{j l} \frac{\rho}{R}\right),
$$

with $j_{l}(x)$ the $l$-order spherical Bessel function of the first kind and $\alpha_{j l}$ its $j$ node. The coefficients $\mathcal{U}_{j l}$ and $\mathcal{V}_{j l}$ (taken as real) obey the standard constraint (see, e.g., Ref. 37)

$$
\mathcal{U}_{j l}^{2}+\mathcal{V}_{j l}^{2}=1
$$

Then, by inserting Eq. (8) into (1a) and (1b), we find the following set of coupled equations (here $E_{j l m}=E_{j l}$ and $\left.\xi_{j l m}=\xi_{j l}\right)$ :

$$
\begin{aligned}
& {\left[E_{j l}-\xi_{j l}\right] \mathcal{U}_{j l}=\Delta_{j l} \mathcal{V}_{j l},} \\
& {\left[E_{j l}+\xi_{j l}\right] \mathcal{V}_{j l}=\Delta_{j l} \mathcal{U}_{j l},}
\end{aligned}
$$

with

$$
\Delta_{j l}=\int_{0}^{R} d \rho \rho^{2} \chi_{j l}^{2}(\rho) \Delta(\rho)
$$

and

$$
\xi_{j l}=\frac{\hbar^{2}}{2 m_{e}} \frac{\alpha_{j l}^{2}}{R^{2}}-\mu .
$$

A nontrivial physical solution of Eqs. (11a) and (11b) exists only when

$$
E_{j l}=\sqrt{\xi_{j l}^{2}+\Delta_{j l}^{2}} \text {. }
$$

The Anderson prescription about the pairing of the timereversed states allows one to rephrase the self-consistency relation [see Eq. (3)] as follows:

$$
\Delta_{j^{\prime} l^{\prime}}=-\sum_{j l}(2 l+1) \frac{M_{j^{\prime} l^{\prime}, j l} \Delta_{j l}}{2 \sqrt{\xi_{j l}^{2}+\Delta_{j l}^{2}}} \tanh \left(\frac{\beta E_{j l}}{2}\right),
$$

where

$$
M_{j^{\prime} l^{\prime}, j l}=-\frac{g}{4 \pi} \int_{0}^{R} d \rho \rho^{2} \chi_{j^{\prime} l^{\prime}}^{2}(\rho) \chi_{j l}^{2}(\rho) .
$$

When deriving Eq. (15), one should keep in mind the property of the spherical harmonics $\sum_{m=-l}^{l}\left|Y_{l m}(\theta, \varphi)\right|^{2}=\frac{2 l+1}{4 \pi}$. We remark that $M_{j^{\prime} l^{\prime}, j l}$ is nothing else but the pairing-interaction matrix element $\left\langle i^{\prime}, \overline{i^{\prime}}|\Phi| i, \bar{i}\right\rangle$ (with $\bar{i}=\{j, l,-m\}$ ) averaged over the states with $m=-l, \ldots, l$ and $m^{\prime}=-l^{\prime}, \ldots, l^{\prime}$, i.e.,

$$
M_{j^{\prime} l^{\prime}, j l}=\frac{1}{\left(2 l^{\prime}+1\right)(2 l+1)} \sum_{m^{\prime}=-l^{\prime}}^{l^{\prime}} \sum_{m=-l}^{l}\left\langle i^{\prime}, \overline{i^{\prime}}|\Phi| i, \bar{i}\right\rangle .
$$

As seen from Eq. (12), a spatially uniform order parameter means that the pairing gaps $\Delta_{j l}$ do not depend on the quantum numbers $j$ and $l$. This is compatible with Eq. (15) only when $M_{j^{\prime} l^{\prime}, j l}$ does not depend on $j^{\prime}$ and $l^{\prime}$. According to the definition given by Eq. (16), we have $M_{j^{\prime} l^{\prime}, j l}=M_{j l, j^{\prime} l^{\prime}}$ and, so, if $M_{j^{\prime} l^{\prime}, j l}$ does not depend on $j^{\prime}, l^{\prime}$, it does not depend on $j, l$ either. So, we arrive at the standard simplified approach of investigating the pairing correlations in metallic grains (see the discussion in the Introduction). Below, we show that the spatial dependence of the order parameter can not be ignored in superconducting nanograins, which implies significant variations of the matrix elements and pairing gaps with the relevant quantum numbers. After a numerical solution of Eq. (15), the position-dependent order parameter can be calculated from

$$
\Delta(\rho)=\sum_{j l} \Delta^{(j l)}(\rho),
$$

with the shell-dependent contribution $\Delta^{(j l)}(\rho)$ given by

$$
\Delta^{(j l)}(\rho)=\frac{g}{8 \pi}(2 l+1) \frac{\chi_{j l}^{2}(\rho) \Delta_{j l}}{\sqrt{\xi_{j l}^{2}+\Delta_{j l}^{2}}} \tanh \left(\frac{\beta E_{j l}}{2}\right) .
$$

\section{DISCUSSION OF RESULTS}

\section{A. Enhanced intrashell matrix elements and quantum-size pinning of the chemical potential}

Numerical calculations were performed with the set of parameters typical for $\operatorname{tin}^{34,38}: \hbar \omega_{D} / k_{B}=195 \mathrm{~K}, g N(0)=0.25$, with $N(0)$ the bulk density of states at the Fermi level [we use the bulk electron density $n_{e}=148 \mathrm{~nm}^{-3}$ (see, e.g., Ref. 39)].

Figure 1(a) shows the critical temperature (in units of the bulk critical temperature $T_{c, B}$ ) versus the nanograin diameter $D$ as calculated from Eq. (15) when the matrix elements of the electron-electron interaction and the size-dependent variation of the chemical potential have been fully taken into account. Results in Fig. 1 are presented for a step $\Delta R=0.01$ $n m$. For each radius, the critical temperature was defined as the temperature above which the spatially averaged order 

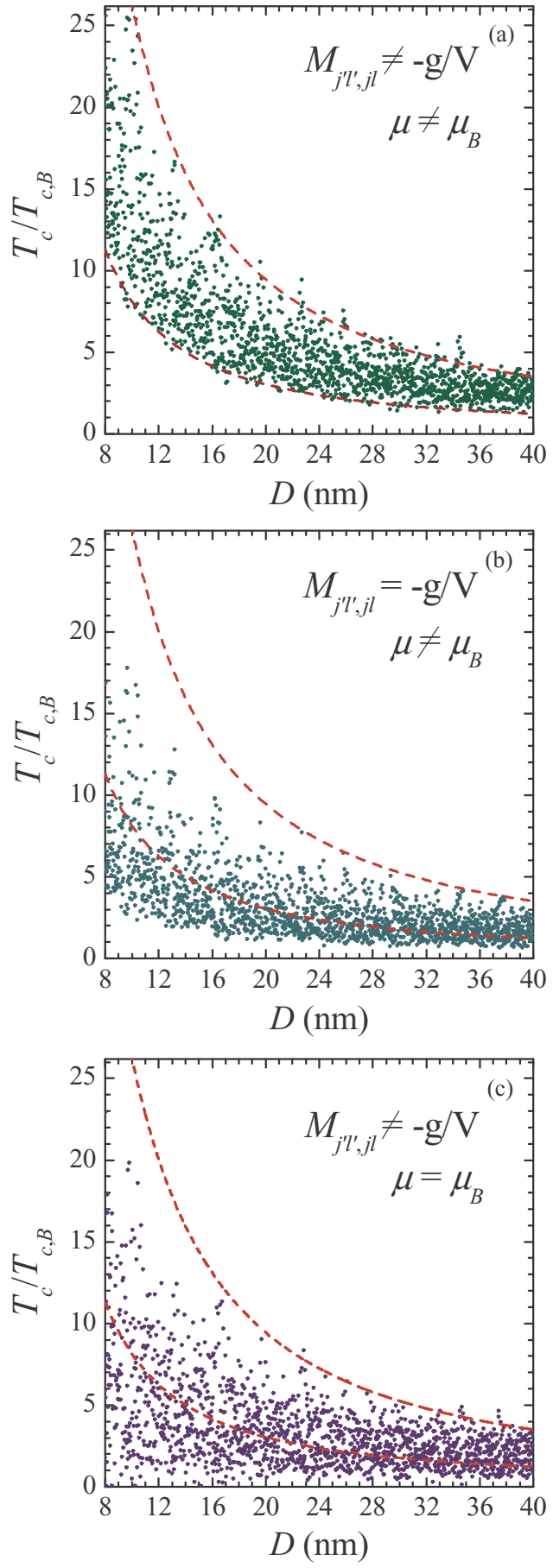

FIG. 1. (Color online) The critical temperature (in units of the bulk critical temperature $T_{c, B}$ ) versus the grain diameter as calculated for (a) $M_{j^{\prime} l^{\prime}, j l} \neq-g / V$ and $\mu \neq \mu_{B}$; (b) $M_{j^{\prime} l^{\prime}, j l}=$ $-g / V$ and $\mu \neq \mu_{B}$; and (c) $M_{j^{\prime} l^{\prime}, j l} \neq-g / V$ and $\mu=\mu_{B}$. The dashed curves in (a) show approximate lower and upper boundaries for the quantum-size oscillations of $T_{c}$, both curves represent the same dependence $T_{c} / T_{c, B}=1+a\left(D_{0} / D\right)^{3 / 2}$, with $D_{0}=$ $50 \mathrm{~nm}$ and $a=1$ (the lower boundary) and $a=3.5$ (the upper one). The same curves are also given in (b) and (c), for comparison.

parameter $\langle\Delta(\rho)\rangle$ becomes smaller than 0.01 of its value at $T=0$. Our numerical results exhibit two features typical of the size-dependent pairing characteristics in high-quality superconducting nanograins. First, we observe an overall increase of $T_{c}$ with decreasing $D$ (it is very pronounced due to the highly symmetric confining geometry). ${ }^{30}$ Second, $T_{c}$ oscillates wildly with $D$. This oscillatory behavior can be understood in the following way. The pair correlations are nonzero only for the states within a finite range (the Debye window) around the chemical potential $\mu$. Moreover, the main contribution to the sum in Eq. (15) comes from the states in the very vicinity of the Fermi level because, in this case, the expression $\Delta_{j l} / \sqrt{\xi_{j l}^{2}+\Delta_{j l}^{2}} \simeq 1\left(\xi_{j l} \simeq 0\right)$. When varying the nanograin size, the number of states in the Debye window changes. The smaller the diameter, the smaller the number of relevant states contributing to the pairing characteristics and, as a result, the more significant is such a change. This change is not monotonous but rather oscillating due to a permanent competition between incoming and outcoming states. As a consequence, all basic pairing characteristics, e.g., $T_{c}$ and pairing gaps $\Delta_{j l}$, exhibit quantum-size oscillations. It is not only typical of nanograins with superconducting correlations (see, e.g., the recent $\operatorname{paper}^{27}$ ), but it is also present in superconducting nanowires ${ }^{12-16}$ and nanofilms. ${ }^{40,41}$ Such oscillations are pronounced for small diameters or thicknesses, but decay with increasing the characteristic size so that $T_{c}$ approaches the bulk critical temperature $T_{c, B}$ (for our parameter $T_{c, B}=4.01 \mathrm{~K}$ ). It is interesting to note that the overall increase of $T_{c}$ with decreasing $D$ in Fig. 1(a) is similar to a size-dependent enhancement of the pairing gap in nuclei, where it is proportional to $1 / \sqrt{A}$ (see, e.g., Ref. 42), with $A$ the number of nucleons. In particular, the two dashed curves in Fig. 1(a) show approximate upper and lower boundaries for $T_{c}$, highlighting the magnitude of the quantum-size oscillations: both curves represent the same dependence, i.e., $T_{c} / T_{c, B}=1+a\left(D_{0} / D\right)^{3 / 2}$, with $D_{0}=$ $50 \mathrm{~nm}$ and $a=1$ and 3.5 for the lower and upper boundaries, respectively $\left[\left(D_{0} / D\right)^{3 / 2} \propto N_{e}^{-1 / 2}\right.$, with $N_{e}=n_{e} V$ the number of electrons]. We remark that real samples exhibit inevitable shape and size fluctuations that affect the high degeneracy of single-electron levels. Hence, measurements on an ensemble of nanograins will significantly smooth the quantum-size oscillations in the critical temperature and reduce its overall enhancement with decreasing nanograin size (see, also, Sec. IV). For instance, in experimentally fabricated tin nanograins of a semispherical shape, the observed enhancement of the excitation gap over its bulk value is about ${ }^{27} 60 \%$ for the particle heights $\approx 10-20 \mathrm{~nm}$. This is significantly smaller than the enhancement of $T_{c}$ shown in Fig. 1(a). However, detailed investigations of the enhancement of $T_{c}$ in superconducting nanograins is beyond the scope of our paper. It is focused on effects of nonuniform pairing, which is of importance even in the presence of shape and size fluctuations and disorder (see the discussion in Sec. IV).

In order to investigate the role of the matrix elements $M_{j^{\prime} l^{\prime}, j l}$ [see Eq. (16)] of the electron-electron interaction, we also show what happens when the true matrix elements are simply replaced by those of the bulklike form: $M_{j^{\prime} l^{\prime}, j l}=$ $-g / V$, which is what is usually done when investigating superconducting correlations in nanograins. The results are displayed in Fig. 1(b) and, as seen, the difference with respect to Fig. 1(a) is significant. To simplify the comparison, we show also in Fig. 1(b) two dashed curves that represent 
TABLE I. Matrix elements $M_{j^{\prime} l^{\prime}, j l}=M_{j l, j^{\prime} l^{\prime}}$ in units of $-g / V$ calculated at $D=7.1 \mathrm{~nm}$ for quantum numbers such that $\xi_{j^{\prime} l^{\prime}}, \xi_{j l}<$ $\hbar \omega_{D}$.

\begin{tabular}{lcccc}
\hline \hline$M_{j^{\prime} l^{\prime}, j l}$ & $j^{\prime}$ & $l^{\prime}$ & $j$ & $l$ \\
\hline 10.62 & 31 & 11 & 31 & 11 \\
1.9 & 31 & 11 & 23 & 29 \\
1.33 & 31 & 11 & 19 & 39 \\
0.64 & 31 & 11 & 8 & 71 \\
0.41 & 31 & 11 & 1 & 101 \\
4.71 & 23 & 29 & 23 & 29 \\
1.7 & 23 & 29 & 19 & 39 \\
0.7 & 23 & 29 & 8 & 71 \\
0.43 & 23 & 29 & 1 & 101 \\
3.72 & 19 & 39 & 19 & 39 \\
0.77 & 19 & 39 & 8 & 71 \\
0.46 & 19 & 39 & 1 & 101 \\
2.69 & 8 & 71 & 8 & 71 \\
0.69 & 8 & 71 & 1 & 101 \\
3.61 & 1 & 101 & 1 & 101 \\
\hline \hline
\end{tabular}

the radius-dependent upper and lower values of $T_{c}$ from Fig. 1(a).

To clarify the physical reason why use of the true matrix elements leads to significant deviations from the results found for $M_{j^{\prime} l^{\prime}, j l}=-g / V$, we show in Table I the numerical values of $M_{j^{\prime} l^{\prime}, j l}$ (calculated in units of $-g / V$ ) for $D=14.2 \mathrm{~nm}$ (only the states within the Debye window are given). As seen, the diagonal (intrashell) matrix elements $M_{j l, j l}$ are strongly enhanced as compared to $-g / V$. However, the matrix elements controlling the scattering of the time-reversed states between different shells (intershell) are often decreased in absolute value with respect to $-g / V$. So, the question arises as to why the superconducting correlations are enhanced for the true matrix elements. The point is that the intershell interactions are of less importance due to a size-dependent pinning of the chemical potential to the groups of degenerate or nearly degenerate levels (shells can be often close to each other in energy) (see the next paragraph). When $\mu$ is pinned to a particular shell, then the single-electron energy measured from $\mu$ is zero for the states from this shell. These states make a major contribution to the superconducting correlations unless diameters are not large enough, $D<20-30 \mathrm{~nm}$, in other words, when the number of contributing shells is less than $10-15$. In this case, the superconducting correlations are nearly determined by the pairing gap $\Delta_{j l}$ associated with the shell pinned to $\mu$. From Eq. (15), it is seen that $\Delta_{j l}$ for the states with $\xi_{j l}=0$ is mainly governed by the intrashell matrix element $M_{j l, j l}$. For instance, when ignoring the contribution of all other states, one simply obtains (at $T=0$ )

$$
\Delta_{j l} \approx-\left(l+\frac{1}{2}\right) M_{j l, j l} .
$$

When the diameter increases beyond 20-30 nm, then the intershell matrix elements approach $-g / V$ while the intrashell matrix elements are still significantly different from the bulklike behavior. However, the role of the states with $\xi_{j l}=0$ is becoming less and less important for larger diameters due to the presence of larger and larger number of shells making a contribution to the pairing correlations. As a consequence, the difference between the data in Figs. 1(a) and 1(b) decreases when approaching $D=35-40 \mathrm{~nm}$, together with the amplitude of the quantum-size oscillations of $T_{c}$.

In the fully self-consistent scheme, the chemical potential is determined in such a way that the mean electron density $n_{e}$ is constant [see Eq. (5)]. However, size-dependent variations of $\mu$ are of importance not only because they simply prevent the mean electron density from deviations. In fact, such deviations are almost insignificant: Our calculations for $\mu=$ $\mu_{B}$ show that $n_{e}$ decreases by a few percent when $D$ is reduced to $10-20 \mathrm{~nm}$. A more interesting thing is that the size-dependent variations of $\mu$ have a pronounced effect on the superconducting correlations. In particular, this can be seen from Fig. 3(c), where $T_{c}$ is calculated for the true matrix elements and $\mu=\mu_{B}$. What is the reason for this suppression of $T_{c}$ ? In the presence of the formation of strongly degenerate electron levels or bunches of electron levels with almost negligible spacing between them, the chemical potential lies mostly at the highest partly filled degenerate level (see, e.g., Refs. 10 and 11). Pairing correlations are significant only within the Debye window around the chemical potential $\mu$ and are strongest ${ }^{28}$ exactly at $\mu$. Hence, when $\mu$ is pinned to a shell level, this favors the pairing correlations at this level and, in turn, through the self-consistency relation, favors the pairing correlations at neighboring shells. In other words, if the level to which the chemical potential is pinned is highly degenerate than the phase space for the strongest pair scattering is enlarged and, consequently, the system gains in interaction energy and, as a result, superconducting correlations are strongly enhanced. It is different when $\mu$ is not pinned to a shell, which is mostly the case for a constant chemical potential, e.g., for $\mu=\mu_{B}$. Here the relevant shells entering the Debye window are as a rule specified by $\xi_{j l} \neq 0$ and, so, their contributions are diminished.

In order to have a better feeling on the effect of the matrix elements and on the pinning of the chemical potential, we also plot $\bar{T}_{c}$, the critical temperature averaged over steps of $1 \mathrm{~nm}$, together with the standard deviation of the critical temperature $\sigma_{T}$ versus the diameter $D$ in Figs. 2(a) and 2(b), respectively. Here, it is clearly seen that the quantumnumber-dependent modifications of the matrix elements and the quantum-size pinning of the chemical potential enhance, on average, the superconducting correlations, enlarging $\bar{T}_{c}$. Note that both above mechanisms make important contribution to $\bar{T}_{c}$ : at diameters $8-30 \mathrm{~nm}$, the averaged critical temperature is significantly reduced when one of them is neglected. In particular, as seen from Fig. 2(a), $\bar{T}_{c}$ is decreased by about a factor of 2.0 at $D \approx 8-10 \mathrm{~nm}$ when either the confinement modification of the matrix elements or the pinning of $\mu$ is switched off. However, the three curves for $\sigma_{T}$ given in Fig. 2(b) are quite close to each other. It means that the amplitude of quantum-size oscillations of $T_{c}$ is not very sensitive to the confinement-induced modifications of the interaction matrix elements and to the size-dependent pinning of $\mu$.

The above discussion is further illustrated by our numerical results for $\mu$ in Fig. 3. As seen from panel (a), when keeping the electron density of the system constant, $\mu$ slightly shifts systematically up with decreasing $D$ and exhibits size-dependent oscillations, as seen from Fig. 3(a). These 

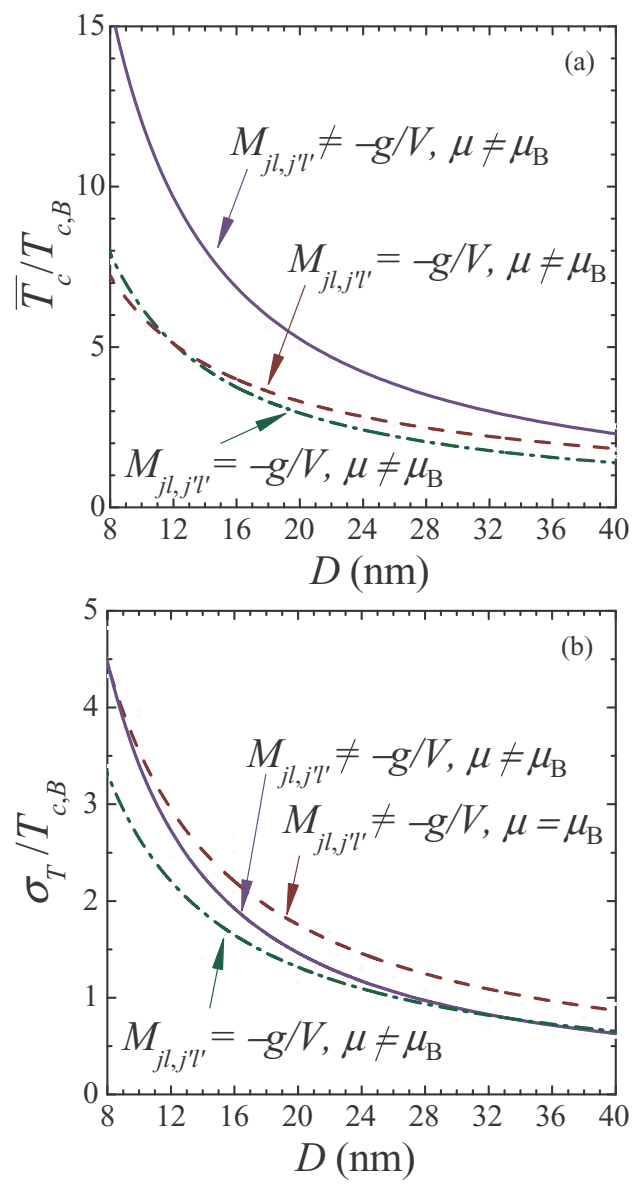

FIG. 2. (Color online) (a) The superconducting critical temperature (in units of $T_{c, B}$ ) averaged over steps of $1 \mathrm{~nm}$ versus the diameter $D$. (b) The standard deviation $\sigma_{T}$ (in units of $T_{c, B}$ ) of the critical temperature as a function of $D$.

oscillations are a signature of the size-dependent pinning of $\mu$ to groups of degenerate or nearly degenerate single-electron levels. This is clearly seen from Fig. 3(b), where variations of $\mu$ (filled squares) are plotted versus $D$ together with the single-electron energies measure from the band bottom, i.e., $\frac{\hbar^{2}}{2 m_{e}} \frac{\alpha_{j l}^{2}}{R^{2}}$ (solid curves). For the sake of simple illustration, panel (b) shows the data for extremely small diameters, where the energy spacing between the shell levels is pronounced. As seen from Fig. 3(b), $\mu$ is in most cases pinned to a shell level, which, as mentioned above, represents incomplete shells. Sometimes $\mu$ can also be found between two neighboring shell levels, which corresponds to the case of a fully occupied lower shell.

\section{B. Spatially nonuniform pair condensate}

In the preceding paragraph, we considered the effect of quantum confinement on pairing correlations in superconducting spherical nanograins through the matrix elements and quantum-size pinning of $\mu$. As discussed at the end of Sec. II, a framework that incorporates both issues appears to be only consistent when the position-dependent superconducting order parameter is taken into account. Thus, our results discussed in the previous section suggest that the spatial variations of
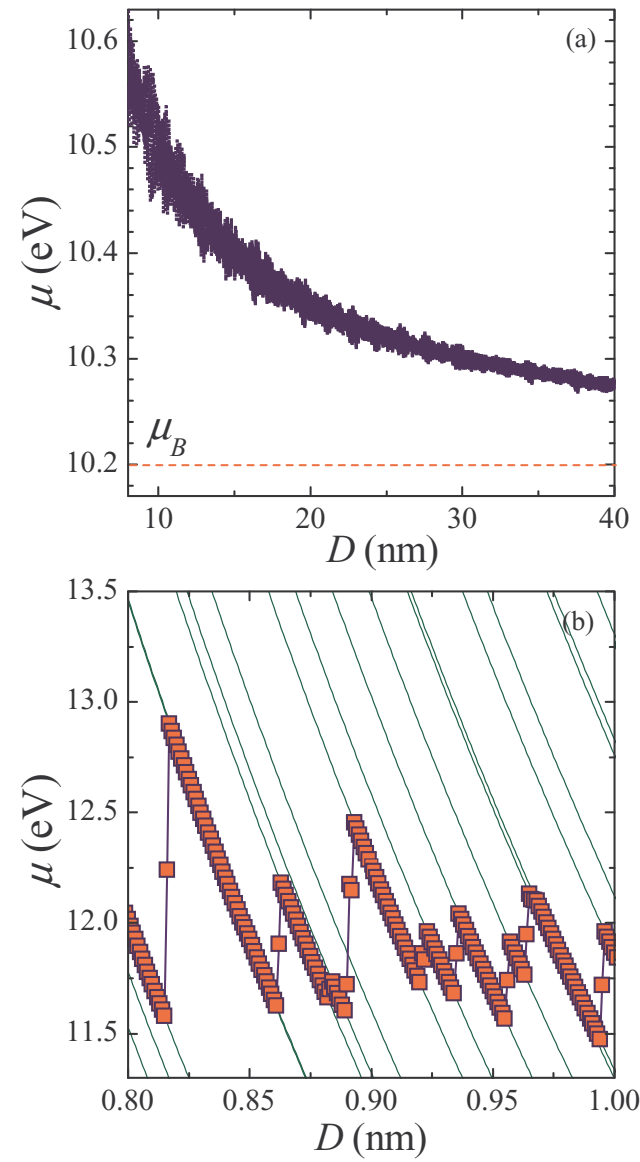

FIG. 3. (Color online) (a) Size variations of the chemical potential. (b) A zoom with details of the quantum-size pinning of the chemical potential: $\mu$ (filled squares) is given together with the single-electron levels (dotted curves) versus $D$. Small diameters are taken for simplicity.

$\Delta(\rho)$ will be pronounced even in nanograins with diameters up to $D=20-30 \mathrm{~nm}$. However, it is usually argued that spatial variations of $\Delta(\rho)$ cost significant extra energy and, so, they are strongly suppressed when $D \ll \xi$, with $\xi$ the bulk coherence length (see, for instance, Ref. 29). In addition, $D$ should be larger than $\lambda_{F}$ : in practice, $k_{F} D \sim 10$ is assumed to be sufficient to ignore any spatial dependence of the order parameter. ${ }^{10,11}$ For typical metallic parameters, $k_{F} D \sim 200$ 400 for $D=10-20 \mathrm{~nm}$ and this is the reason why the spatial dependence of the order parameter was ignored in most papers on superconducting correlations in nanograins. Below, we will critically examine spatial variations of $\Delta(\rho)$ and will show that the above criterion for nonuniform pairing has to be revisited.

In Fig. 4, the radial dependence of the superconducting order parameter is shown as calculated from Eq. (16) for $D=12 \mathrm{~nm}$ (a), $13.52 \mathrm{~nm}$ (b), $14.2 \mathrm{~nm}$ (c), $16 \mathrm{~nm}$ (d), $16.4 \mathrm{~nm}(\mathrm{e})$, and $17.54 \mathrm{~nm}$ (f). The shells making a contribution to the superconducting correlations are also displayed in each panel, and the quantum numbers of the shell level pinned to $\mu$ are underlined. As seen, we in general have a nonuniform distribution of the pair condensate for diameters $D=10-20 \mathrm{~nm}$, which is in agreement with our expectations. For example, let us consider the results plotted in panel 4(c). Here, $\mu$ is pinned to the shell level $(l, j)=(101,1)$ and, 

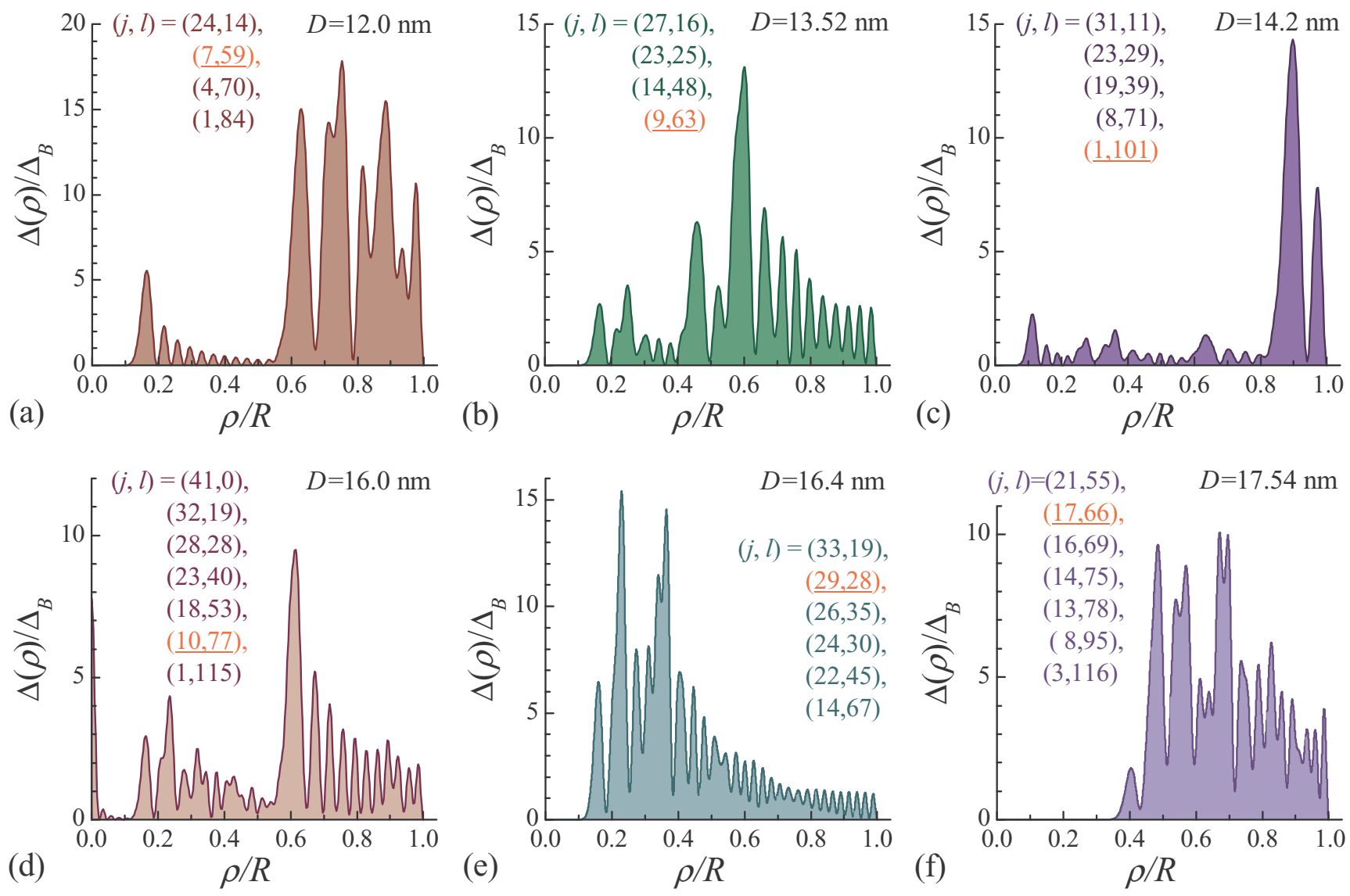

FIG. 4. (Color online) Spatial distribution of the pair condensate in spherical nanograins: $\Delta(\rho)$ (calculated at $T=0$ ) versus $\rho$ for diameters (a) $D=12 \mathrm{~nm}$, (b) $13.52 \mathrm{~nm}$, (c) $14.2 \mathrm{~nm}$, (d) $16 \mathrm{~nm}$, (e) $16.4 \mathrm{~nm}$, (f) and $17.54 \mathrm{~nm}$.

so, single-electron states with $j=1$ and $l=101$ make a major contribution to $\Delta(\rho)$, which results in a significant enhancement of the order parameter next to the edge, i.e., for $\rho / R=0.9-1.0$. The profile of this enhancement is determined by the radial wave function $\chi_{1,101}^{2}(\rho)$ with two pronounced local maxima $\left(\Delta / \Delta_{B}=14.3\right.$ and 7.2 at $\rho / R=0.9$ and 0.97 , respectively) and one node (recall that $j$ is the number of the nodes of the radial wave function). All the other shells displayed in Fig. 4(c) are specified by $\xi_{j l} \neq 0$ and, as a result, their contribution is much less significant. The local maximum $\Delta(\rho) / \Delta_{B}=2.3$ at $\rho / R=0.1$ is due to states $(j, l)=(31,11)$. The shells with $(j, l)=(23,29)$ and $(19,29)$ are responsible for local enhancements of the order parameter up to $1.5-2.0 \Delta_{B}$ at $\rho / R=0.27$ and 0.36 , respectively. At last, the shell $(8,71)$ produces the local maximum at $\rho / R=0.64$. In general, the larger the angular momentum, the larger the values of $\rho / R$ at which the corresponding single-electron states have an effect on the profile of $\Delta(\rho)$.

It is worth noting that, typically, the order parameter is strongly suppressed in the center $(\rho=0)$ except in rare cases when states with zero angular momentum contribute to the pairing correlations. One such example is given in Fig. 4(d), where a narrow peak can be seen at $\rho=0$ due to the contribution of the states with $(j, l)=(41,0)$.

From Fig. 4, it follows that the radial distribution of the pair condensate remains strongly nonuniform even for $D \approx 20 \mathrm{~nm}$. We would like to note that, when selecting concrete values of $D$ for Fig. 4, we did not take diameters for which $T_{c}$ is close to the upper dashed curve in Fig. 1(a). In the case of a strong enhancement of $T_{c}$, the radial distribution of the pair condensate is, as a rule, strongly nonuniform. The points selected for Fig. 4 are mainly in the vicinity of the lower dashed curve in panel (a) of Fig. 1: for $D=13.52,14.2,16$, and $16.4 \mathrm{~nm}$, we have $T_{c} / T_{c, B}=6.41,6.48,4.082$, and 3.78, respectively. However, even in this case, the order parameter can vary with position by an order of magnitude. Spatial variations of $\Delta(\rho)$ are significantly relaxed only when $D$ approaches $30-40 \mathrm{~nm}$, as seen from Fig. 5 .

For our parameters, $k_{F}=16.4 \mathrm{~nm}$ and, so, we obtain $k_{F} D \approx 300$ for $D \approx 20 \mathrm{~nm}$. Hence, the criterion $k_{F} D \gg 1$ is not very useful in order to estimate the effect of spatial variations of the pair condensate. Based on our numerical study, we suggest another criterion related to a more sensitive energy scale, which is governed by the bulk pairing gap $\Delta_{B}$. The spatial distribution of the order parameter is always strongly inhomogeneous when $\delta \sim \Delta_{B}$ (here it is even better to replace $\Delta_{B}$ by the size-dependent pairing gap). The spatial variations decay with a decrease in the ratio of the mean interlevel spacing to the bulk order parameter, i.e., $\delta / \Delta_{B}$, and our numerical results suggest that such variations are significantly reduced only when $\delta / \Delta_{B}<0.05-0.1$ (recall that effects of a magnetic field are beyond the scope of our paper). For $\mathrm{Sn}$ spherical superconducting grains, this regime is achieved when $D>40-50 \mathrm{~nm}$ [note that $\delta \approx 2 \pi^{2} \hbar^{2} /\left(m k_{F} V\right)$ 

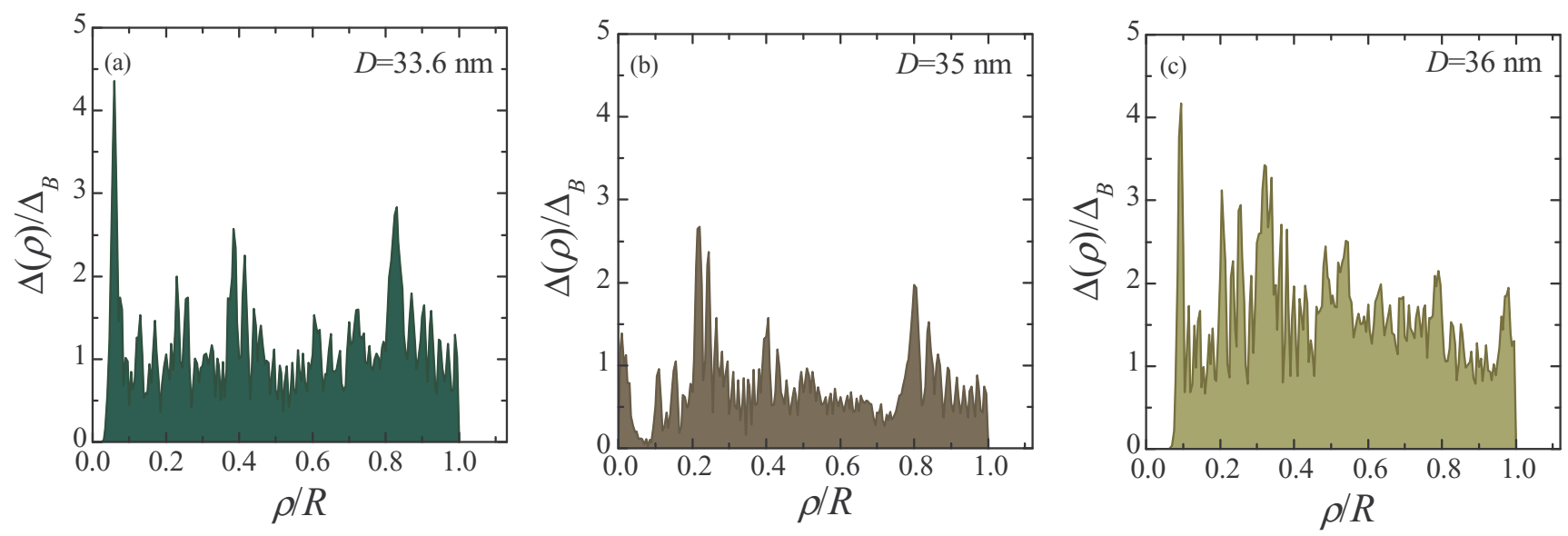

FIG. 5. (Color online) The order parameter $\Delta(\rho)$ for diameters (a) $D=33.6 \mathrm{~nm}$, (b) $D=35 \mathrm{~nm}$, and (c) $D=36 \mathrm{~nm}$.

underestimates the intershell spacing for spherical confining potential]. Despite that our results are for a highly symmetric confining geometry, we can expect that the order parameter will be always spatially nonuniform for $\delta / \Delta_{B}>0.1-0.2$, even when shape imperfections and disorder nearly dissolve the shell structure. The reason is that the number of contributing states [i.e., the states in the energy interval $\approx\left(\mu-\Delta_{B}, \mu+\right.$ $\left.\Delta_{B}\right)$ ] is not very large for $\delta / \Delta_{B}>0.1$. In this case, the states pinned to $\mu$ always make a major contribution to the order parameter and, so, the profile of the squared absolute value of the corresponding wave function will mainly determine the spatial distribution of the condensate. Thus, the domain $\delta / \Delta_{B}>0.1-0.2$ is in general characterized by strong effects due to spatially nonuniform pairing. We note that this conclusion is in agreement with the results of Refs. 17 and 32, where it was found that the energy dependence of the relevant matrix elements becomes more pronounced with increasing the ratio $\delta / \Delta_{B}$. For $\delta / \Delta_{B}>0.1-0.2$, the pairing in small metallic superconducting grains becomes similar to that in a superfluid Fermi gas in a harmonic-oscillator trap, where the spatial distribution of the superfluid condensate is in general spatially inhomogeneous except in a small region in the center of the trap (for more details, see, Ref. 43 about shell effects, Ref. 44 about the pairing of the time-reversed states and the relevant corrections, and Ref. 45 about the size-dependent oscillations of the superfluid properties).

We remark that our results do not contradict the usual argument that spatial variations of the order parameter cost extra energy. Let us compare a bulk superconductor with a superconducting nanograin. In bulk, the relevant matrix elements controlling the scattering of the time-reversed states are $-g / V$ and the order parameter is spatially uniform (in the absence of a magnetic field). As opposed to bulk, the pair condensate significantly varies with position in nanograins, which results, of course, in an increase of the kinetic energy. However, the intrashell matrix elements are now enhanced in absolute value as compared to $-g / V$ due to quantum confinement. This compensates the energy cost due to spatial variations of the order parameter.

The discussion in the preceding paragraph is also related to arguments that are invoked in the conventional GinzburgLandau theory. According to these arguments, the order parameter is uniform in samples with size smaller than the bulk coherence length. When applying this to nanograins, one can conclude that the pair condensate should not vary with its position. However, this is not true. It is well known that one should be careful when applying conventional GinzburgLandau theory to superconductors with characteristic size smaller than the zero-temperature (BCS) coherence length $\xi_{0}$. Strictly speaking, Gor'kov's derivation of the conventional Ginzburg-Landau formalism from the BCS approach is not applicable on a scale smaller than $\xi_{0}$ (see, e.g., Ref. 38). For Sn, we have $\xi_{0} \approx 230 \mathrm{~nm}$ (see, e.g., Ref. 34). Thus, in the case of interest $D \ll \xi_{0}$, and one can hardly invoke conventional Ginzburg-Landau formalism to check whether or not $\Delta(\rho)$ varies with $\rho$.

\section{Confinement-induced Andreev-type states}

Here, we would like to discuss one more important issue related to spatially nonuniform pairing in nanograins. This is the formation of Andreev-type states induced by quantum confinement ${ }^{14,49}$ (see also a similar paper ${ }^{45}$ discussing Andreev-type states in ultracold trapped superfluid Fermi gases). Since the 1960s (see Refs. 46-48), it is known that quasiparticles can "feel" a spatial variation of the superconducting order parameter as a kind of potential barrier. This physical mechanism (referred to as the Andreev mechanism below) is the basis for Andreev quantization investigated previously for the core of a single vortex for the mixed state of a typeII superconductor ${ }^{47}$ and for an isolated normal region of the intermediate state of a type-I superconductor ${ }^{48}$ (or for a similar case of SNS contacts $\left.{ }^{46}\right)$. Based on our discussion in Sec. III B, one can expect that Andreev-type states can play a remarkable role in superconducting nanograins due to significant spatial variations of the superconducting order parameter. This is very similar to recently investigated Andreev-type states in superconducting nanowires and nanofilms, ${ }^{14,49}$ where the pair condensate is position dependent in the direction perpendicular to the nanowire and nanofilm due to the quantization of the perpendicular electron motion. In Ref. 49, it was shown that

$$
\Delta_{i}=\int d^{3} r \Delta(\mathbf{r})\left[\left|u_{i}(\mathbf{r})\right|^{2}+\left|v_{i}(\mathbf{r})\right|^{2}\right],
$$



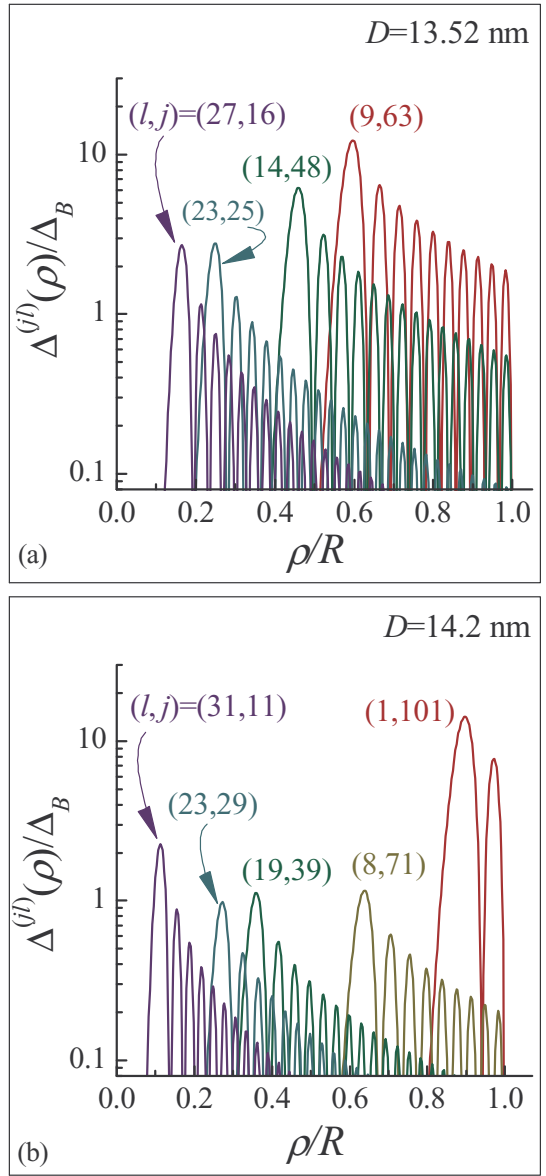

FIG. 6. (Color online) Shell-dependent contributions to the order parameter $\Delta^{(j l)}(\rho)$ for relevant shells: (a) $D=13.52 \mathrm{~nm}$, $(j, l)=(27,16),(23,25),(14,48)$, and $(9,63)$; (b) $D=14.2 \mathrm{~nm}$, $(j, l)=(31,11),(23,29),(19,39),(8,71)$, and $(1,101)$.

which means that the pairing energy gap $\Delta_{i}$ is the averaged value of the order parameter "watched" by the quasiparticles with quantum numbers $i$. Note that $\left|u_{i}(\mathbf{r})\right|^{2}+\left|v_{i}(\mathbf{r})\right|^{2}$ can be interpreted as the spatial distribution of quasiparticles according to the well-known constraint $\int d^{3} r\left(\left|u_{i}(\mathbf{r})\right|^{2}+\left|v_{i}(\mathbf{r})\right|^{2}\right)=1$ [see, e.g., Ref. 37 and Eq. (10)]. When inserting Eqs. (6) into Eq. (18), one can easily obtain Eq. (12) with $\Delta_{i}=\Delta_{j l}$. If quasiparticles avoid the domains of enhanced pair condensate, the corresponding integral in the right-hand side of Eq. (18) becomes smaller and, hence, such quasiparticles have smaller pairing gaps $\Delta_{j l}$. They can be referred to as Andreev-type states.

Our numerical study of quantum-number-dependent pairing gaps $\Delta_{j l}$ for metallic nanograins reveals a significant role of the Andreev mechanism. Let us consider $D=13.52 \mathrm{~nm}$, the corresponding spatial distribution of the pair condensate given in Fig. 4(b). To show how different species of quasiparticles are distributed in the radial direction in this case, we plotted in Fig. 6(a) the radial-dependent shell contributions (at $T=0$ ) $\Delta^{(j l)}(\rho)$ [see Eqs. (16) and (17)]. We remark that such a representation is more informative than simply a plot of $\left|u_{j l}(\rho)\right|^{2}+\left|v_{j l}(\rho)\right|^{2}$. First, the radial dependence of $\Delta^{(j l)} \propto$ $\chi_{j l}^{2}(\rho)$ is the same as that of $\left|u_{j l}(\rho)\right|^{2}+\left|v_{j l}(\rho)\right|^{2} \propto \chi_{j l}^{2}(\rho)$ [see Eq. (8)]. Second, a plot of $\Delta^{(j l)}(\rho)$ gives also information as to how the corresponding states contribute to $\Delta(\rho)$. From Fig. 4(c), we can see that a significant enhancement of the order parameter occurs at $\rho / R=0.45-0.7$. From Fig. 6(a), it is clear that this enhancement is due to the states with $(j, l)=(14,48)$ and $(9,63)$. Other shells, i.e., $(27,16)$ and $(23,25)$ contribute less, and the corresponding quasiparticles, representing Andreev-type states, are mainly located beyond the domain $\rho=0.45-1.0$. As a result, they have smaller pairing gaps, i.e., $\Delta_{27,16}=2.65 \Delta_{B}$ and $\Delta_{23,25}=2.81 \Delta_{B}$, as compared to $\Delta_{14,48}=4.098 \Delta_{B}$ and $\Delta_{9,63}=5.77 \Delta_{B}$. As seen, the quasiparticles with $(j, l)=(27,16)$ are most successful in avoiding the local enhancement of $\Delta(\rho)$ at $\rho / R=0.45-0.7$ and, so, $\Delta_{27,16}$ is the smallest pairing gap. Such a manifestation of Andreev mechanism is not a particular feature of $D=13.52 \mathrm{~nm}$. In general, $\Delta_{j l}$ strongly varies with $j$ and $l$ for diameters $<30-40 \mathrm{~nm}$, i.e., where spatial variations of the order parameter are still pronounced. Quite often, such variations can be an order of magnitude as, e.g., for $D=14.2 \mathrm{~nm}$ [see $\Delta(\rho)$ given in Fig. 3(c)]. At this diameter, a great enhancement of $\Delta(\rho)$ takes place at $\rho / R=0.9$. This is due to the contribution of the shell with $(j, l)=(1,101)$ [see Fig. 6(b)]. Other shells make much less important inputs and the corresponding quasiparticles are mainly distributed beyond the domain $\rho / R=0.9-1.0$. So, as compared to $\Delta_{1,101}=$ $9.32 \Delta_{B}$, they have significantly smaller pairing gaps, i.e., $\Delta_{31,11}=1.6 \Delta_{B}, \Delta_{23,29}=1.62 \Delta_{B}, \Delta_{19,39}=1.72 \Delta_{B}$, and $\Delta_{8,71}=2.35 \Delta_{B}$. Thus, the interplay of Andreev mechanism and quantum confinement is responsible for variations of $\Delta_{j l}$ with the relevant quantum numbers.

One could expect that such a serious difference in pairing gaps of different quasiparticle species can result in a pronounced drop of the ratio of $\Delta_{E}$ (the minimal energy gap) to the critical temperature $k_{B} T_{c}$, similar to the case for superconducting quantum nanowires. ${ }^{14}$ The main idea here is that $\Delta_{E}$ is governed by Andreev-type states and, hence, is decreased. Unlike $\Delta_{E}, T_{c}$ is controlled by the quasiparticles making a major contribution to $\Delta(\rho)$ and, so, $T_{c}$ is coupled to their higher pairing gaps. As a result, $\Delta_{E} / k_{B} T_{c}$ can be significantly smaller than in bulk. For instance, one can expect that $\Delta_{E}=\Delta_{31,11}=1.6 \Delta_{B}$ at $D=14.2 \mathrm{~nm}$, while $T_{c}$ is governed by $\Delta_{1,101}=9.32 \Delta_{B}$. However, this is not correct for nanograins. The point is that $\Delta_{E}$ is a spectroscopical gap, which is probed by STM. It is defined as $\Delta_{E}=\min _{j l} E_{j l}$. For nanowires, the subband-dependent pairing gap is always the minimal quasiparticle energy due to a quasifree spectrum in the direction parallel to the nanowire. For nanograins, this is different. In particular, for $D=14.2 \mathrm{~nm}$, we have the following single-electron energies (absorbing $\mu$ ) of the relevant shells: $\xi_{31,11}=-18.6 \Delta_{B}, \xi_{23,29}=-26.03 \Delta_{B}$, $\xi_{19,39}=20.9 \Delta_{B}, \quad \xi_{8,71}=22.6 \Delta_{B}$, and $\xi_{1,101}=0$. Hence, one can calculate that $\Delta_{E}=E_{1,101}=\Delta_{1,101}$ in spite of the fact that $\Delta_{1,101}$ is the largest pairing gap. Thus, although the Andreev mechanism results in multiple-gap structure in superconducting nanograins, its role is shadowed by the nonzero interlevel spacing.

\section{CONCLUSIONS AND DISCUSSION}

In conclusion, we have shown that the spatial distribution of the pair condensate is essentially nonuniform in metallic 
nanograins. In particular, the spatially nonuniform pairing can proliferate in nanograins even when $k_{F} D \sim 300$ and, so, the usual criterion to neglect variations of the superconducting condensate with position, i.e., $k_{F} D \gg 1$, is not very useful and can result in wrong conclusions. This is the reason why effects due to spatially nonuniform pairing in superconducting grains were previously overlooked. Our study suggests that a new criterion should be based on a more delicate energy scale (as compared to the Fermi energy), which, in the superconducting state, is given by the bulk order parameter $\Delta_{B}$. It turns out that the pairing becomes spatially nonuniform when the interlevel spacing $\delta$ exceeds $0.1-0.2 \Delta_{B}$. Variations of the order parameter with position exhibit a pronounced enhancement with an increase of $\delta / \Delta_{B}$. When $\delta \sim \Delta_{B}$, such variations can be almost an order of magnitude in highly symmetric grains. At first sight, this seems impossible because it costs extra energy for such spatial variations. However, a nonuniform distribution of the pair condensate is accompanied by enhanced pairing-interaction matrix elements, which compensate the energy cost for an inhomogeneous distribution of the condensate. Another point is the size-dependent pinning of the chemical potential to groups of degenerate or nearly degenerate energy levels. Such a pinning plays the role of a filter that increases the contribution of the single-electron levels in the vicinity of the chemical potential and suppresses contributions of other states. This results in an additional mechanism favoring spatially nonuniform pairing in metallic nanograins.

In this paper, we investigated a highly symmetric confining geometry. Due to this feature, the problem becomes effectively one dimensional (the order parameter depends only on the radial coordinate) and, so, sufficiently large diameters up to $D \approx 40 \mathrm{~nm}$ can be investigated. This size is almost impossible to reach theoretically for grains with the order parameter depending on three relevant coordinates due to time-consuming numerical calculations. Such an effectively one-dimensional problem has large degeneration factors for the corresponding shell structure, resulting in a significant enhancement of the pairing correlations. In reality, there can be several issues that may lead to a splitting of the shell levels. It will decrease the degeneracy factors and, so, reduce the pairing correlations since the main contribution to the sum in the gap equation comes from the transitions within the same shell pinned to the chemical potential. Among such issues is the Jahn-Teller deformation, i.e., the transformation of a spherical nanograin with incompletely filled shells to an ellipsoidal shape. In addition, the surface imperfections and impurities can significantly change the distribution of single-electron levels. However, our qualitative results are quite generic and do not depend on a particular shape of the nanograin and the presence of possible imperfections. For instance, when $\delta \sim \Delta_{B}$, the pair condensate will always be spatially nonuniform because only a few single-electron levels enter the energy interval $\approx\left[\mu-\Delta_{B}, \mu+\Delta_{B}\right]$. Due to the dominant contribution of such levels to $\Delta(\mathbf{r})$, one can expect that the pair condensate acquires a profile governed by the squared absolute value of the wave function for the single-electron state closest in energy to $\mu$. This is significantly strengthened by an increase (in absolute value) of the diagonal matrix elements $\langle i, \bar{i}|\Phi| i, \bar{i}\rangle$ and, in addition, by the pinning of the chemical potential to the single-particle levels.

We remark that the diagonal matrix elements, i.e., $\langle i, \bar{i}|\Phi| i, \bar{i}\rangle$ [see the definition for $\Phi$ below Eq. (3)] are always enhanced as compared to $-g / V$ in the presence of quantum confinement, whatever disorder and shape imperfections. This can be seen from the following simple arguments. By
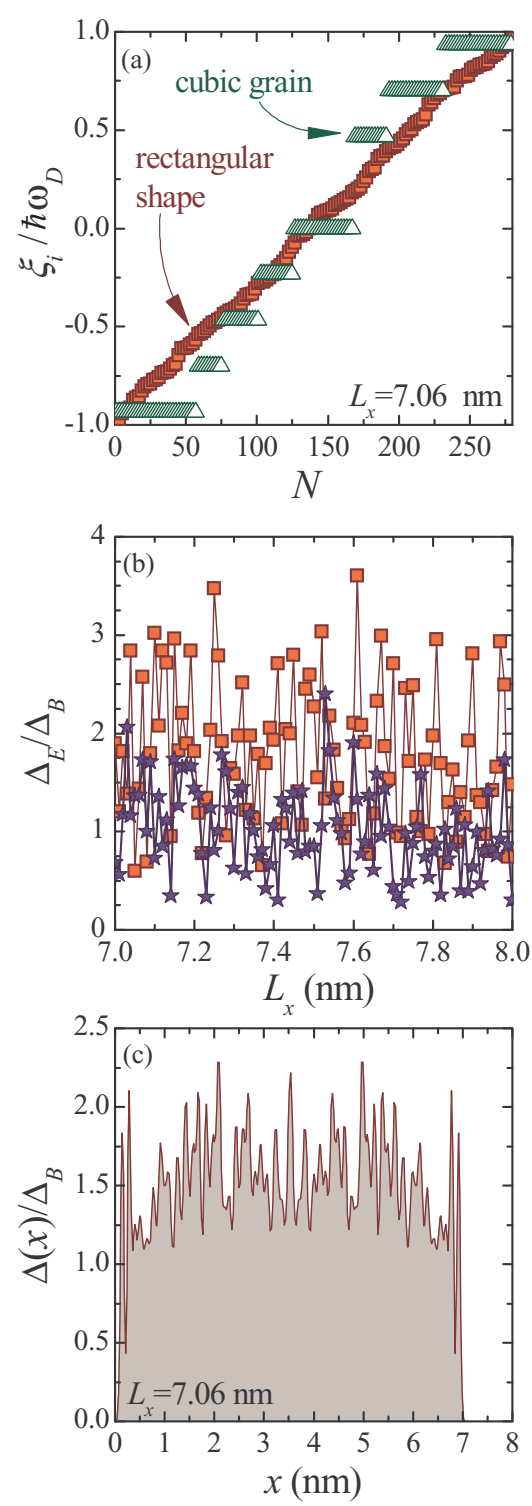

FIG. 7. (Color online) (a) Single-electron energies $\xi_{i}$ (given in units of the Debye energy $\hbar \omega_{D}$ ) ordered in ascending manner versus the ordering number $N$ for the rectangular-shaped aluminum nanograin with $L_{x}=7.06 \mathrm{~nm}, L_{y}=L_{x} / 1.1, L_{z}=1.1 L_{x}$ (squares) and for a cubic nanograin with $L_{x}=7.06 \mathrm{~nm}$ (triangles). (b) The size-dependent excitation gap $\Delta_{E} / \Delta_{B}$ versus $L_{x}$ (in steps of $\delta L_{x}=$ $0.01 \mathrm{~nm}$ ) for an aluminum nanograin of the rectangular shape with the dimensions $L_{x}, L_{y}=L_{x} / 1.1, L_{z}=1.1 L_{x}$ : squares represent the results calculated with the modified matrix elements and with proper variations of $\mu$; stars are the data obtained for the bulklike matrix elements $-g / V$ and $\mu=\mu_{B}$. (c) The spatial distribution of the pair condensate in the rectangular grain with $L_{x}=7.06 \mathrm{~nm}$ and $L_{y}=L_{x} / 1.1, L_{z}=1.1 L_{x}, \Delta(x)=\left.\Delta(x, y, z)\right|_{y=x / 1.1, z=1.1 x}$. 
introducing $\varphi_{i}(\mathbf{r})$, the wave function associated with state $i$, one can write

$$
\langle i, \bar{i}|\Phi| i, \bar{i}\rangle=-g \int d^{3} r\left|\varphi_{i}(\mathbf{r})\right|^{4} .
$$

Due to the normalization condition, we have $\left|\varphi_{i}(\mathbf{r})\right|^{2}=\frac{1}{V}+$ $d_{i}(\mathbf{r})$, where $\int d^{3} r d_{i}(\mathbf{r})=0$. Then, the above matrix element can be rearranged as

$$
\langle i, \bar{i}|\Phi| i, \bar{i}\rangle=-\frac{g}{V}\left[1+V \int d^{3} r d_{i}^{2}(\mathbf{r})\right] .
$$

The second term in square brackets is always positive in the presence of quantum confinement, i.e., when $d_{i}(\mathbf{r}) \neq 0$. It is zero only when $\varphi_{i}(\mathbf{r})$ 's are chosen in the form of plane waves, which results in $\langle i, \bar{i}|\Phi| i, \bar{i}\rangle=-g / V$.

The above discussion can be supplemented by our numerical results calculated from the BCS-type equation similar to Eq. (15), but now for aluminum nanograins of rectangular shape with dimensions $L_{x}, L_{y}=L_{x} / 1.1, L_{z}=1.1 L_{x}$. For aluminum, we have $34,38,39 \hbar \omega_{D} / k_{B}=375 \mathrm{~K}, g N(0)=0.18$, and $\mu_{B}=11.67 \mathrm{eV}$, which corresponds to the electron density $n_{e}=181 \mathrm{~nm}^{-3}$. In Fig. 7(a), single-electron levels arranged in ascending order are shown within the Debye window for the rectangular nanograin with $L_{x}=7.06 \mathrm{~nm}$ (squares). The same is also given here for a cubic aluminum nanograin with $L_{x}=L_{y}=L_{z}=7.06 \mathrm{~nm}$ (triangles). As seen, singleelectron levels for rectangular shape are distributed in a nearly equidistant manner (with $\delta \approx 0.2-0.3 \mathrm{meV} \sim \Delta_{B}$ ) contrary to the states in case of cubic geometry. It is well known that an almost equidistant distribution ${ }^{4}$ of single-electron levels near $\mu$ is also expected in the presence of significant imperfections such as surface roughness and/or impurities. So, our results in Fig. 7 give a feeling about the role of the spatially nonuniform pairing in disordered metallic grains. The excitation energy gap $\Delta_{E}$ for the rectangular nanograin is shown in units of $\Delta_{B}$ in Fig. 7 as a function of $L_{x}$ in the interval $L_{x}=7-8 \mathrm{~nm}$. Here, squares represent our results calculated with the modification of the matrix elements and with $\mu$ varying with $L_{x}$; stars are the results found for the bulklike matrix elements $-g / V$ and $\mu=\mu_{B}$. As seen, $\Delta_{E}\left(\propto T_{c}\right)$ is now two times larger as compared to $\Delta_{B}$ (on average), which is much less significant than for highly symmetric grains (compare with Fig. 1) due to a splitting of the shell levels. However, the effect of interest is still pronounced: $\Delta_{E}$ calculated for the modified matrix elements and with account of size variations of $\mu$ is generally larger by a factor of 1.5-2.0. The spatial profile of the order parameter is nonuniform with local enhancements over its average value by about $100 \%$ [see, e.g., Fig. 7(c)]. For rectangular grains with $L_{x}=7-8 \mathrm{~nm}$, we have $\delta \sim \Delta_{B}$. However, as we checked, the spatially nonuniform pairing and the related effects of the modification of the relevant matrix elements and the size-dependent pinning of $\mu$ are significant even for smaller $\delta$ 's, i.e., when $\delta>0.1-0.2 \Delta_{B}$ $\left(L_{x}<14-15 \mathrm{~nm}\right)$. For instance, for $L_{x}=11 \mathrm{~nm}$, the order parameter exhibits variations of about $30 \%-40 \%$ of its averaged value. These results are in agreement with our expectations based on the investigation of the highly symmetric spherical grains.

We also note that STM can hardly provide us with any information about spatial variations of the pair condensate in the center of a superconducting grain. However, STM can measure the order parameter next to the grain surface. If a grain is not symmetric and the order parameter significantly varies with position, such a measurement might produce results depending on the point of the surface.

\section{ACKNOWLEDGMENTS}

This work was supported by the Alexander von Humboldt Foundation, the Flemish Science Foundation (FWO-Vl), and the Belgian Science Policy (IAP). M.D.C. acknowledges support of the European Community under a Marie Curie IEF Action (Grant Agreement No. PIEF-GA-2009-235486ScQSR).
${ }^{*}$ Current address: Université Bordeaux I, LOMA, FR-33405 Talence Cedex, France.

${ }^{1}$ R. Parmenter, Phys. Rev. 166, 392 (1968).

${ }^{2}$ J. M. Blatt and C. J. Thompson, Phys. Rev. Lett. 10, 332 (1963).

${ }^{3}$ B. Mühlschlegel, D. J. Scalapino, and R. Denton, Phys. Rev. B 6, 1767 (1972).

${ }^{4}$ R. A. Smith and V. Ambegaokar, Phys. Rev. Lett. 77, 4962 (1996).

${ }^{5}$ A. Perali, A. Bianconi, A. Lanzara, and M. L. Saini, Solid State Commun. 100, 181 (1996).

${ }^{6}$ F. Braun and J. von Delft, Phys. Rev. B 59, 9527 (1999).

${ }^{7}$ G. Sierra, J. Dukelsky, G. G. Dussel, J. von Delft, and F. Braun, Phys. Rev. B 61, R11890 (2000).

${ }^{8}$ V. N. Gladilin, V. M. Fomin, and J. T. Devreese, Solid State Commun. 121, 519 (2002).

${ }^{9}$ E. A. Yuzbashyan, A. A. Baytin, and B. L. Altshuler, Phys. Rev. B 68, 214509 (2003).

${ }^{10}$ Y. N. Ovchinnikov and V. Z. Kresin, Eur. Phys. J. B 45, 5 (2005); 47, 333 (2005).
${ }^{11}$ V. Z. Kresin and Y. N. Ovchinnikov, Phys. Rev. B 74, 024514 (2006).

${ }^{12}$ A. A. Shanenko and M. D. Croitoru, Phys. Rev. B 73, 012510 (2006).

${ }^{13}$ M. D. Croitoru, A. A. Shanenko, and F. M. Peeters, Phys. Rev. B 76, 024511 (2007).

${ }^{14}$ A. A. Shanenko, M. D. Croitoru, R. G. Mints, and F. M. Peeters, Phys. Rev. Lett. 99, 067007 (2007).

${ }^{15}$ A. A. Shanenko, M. D. Croitoru, and F. M. Peeters, Phys. Rev. B 78, 024505 (2008).

${ }^{16}$ M. D. Croitoru, A. A. Shanenko, C. C. Kaun, and F. M. Peeters, Phys. Rev. B 80, 024513 (2009).

${ }^{17}$ A. M. García-García, J. D. Urbina, E. A. Yuzbashyan, K. Richter, and B. L. Altshuler, Phys. Rev. Lett. 100, 187001 (2008).

${ }^{18}$ N. K. Kuzmenko and V. M. Mikhajlov, Phys. C (Amsterdam) 470, 193 (2010).

${ }^{19}$ I. Giaever and H. R. Zeller, Phys. Rev. Lett. 20, 1504 (1968); H. R. Zeller and I. Giaever, Phys. Rev. 181, 789 (1969). 
${ }^{20}$ W.-H. Li, C. C. Yang, F. C. Tsao, S. Y. Wu, P. J. Huang, M. K. Chung, and Y. D. Yao, Phys. Rev. B 72, 214516 (2005).

${ }^{21}$ W.-H. Li, C.-W. Wang, C.-Y. Li, C. K. Hsu, C. C. Yang, and C.-M. Wu, Phys. Rev. B 77, 094508 (2008).

${ }^{22}$ S. Bose, P. Raychaudhuri, R. Banerjee, P. Vasa, and P. Ayyub, Phys. Rev. Lett. 95, 147003 (2005).

${ }^{23}$ S. Bose, C. Galande, S. Chockalingam, R. Banerjee, P. Raychaudhuri, and P. Ayyub, J. Phys. Condens. Matter 21, 205702 (2009).

${ }^{24}$ D. C. Ralph, C. T. Black, and M. Tinkham, Phys. Rev. Lett. 74, 3241 (1995).

${ }^{25}$ C. T. Black, D. C. Ralph, and M. Tinkham, Phys. Rev. Lett. 76, 688 (1996).

${ }^{26}$ I. Brihuega, S. Bose, M. M. Ugeda, C. H. Michaelis, and K. Kern, e-print arXiv:0904.0354.

${ }^{27}$ S. Bose, A. M. García-García, M. M. Ugeda, J. D. Urbina, C. H. Michaelis, and K. Kern, Nat. Mater. 9, 550 (2010).

${ }^{28}$ J. von Delft, A. D. Zaikin, D. S. Golubev, and W. Tichy, Phys. Rev. Lett. 77, 3189 (1996).

${ }^{29}$ K. A. Matveev and A. I. Larkin, Phys. Rev. Lett. 78, 3749 (1997).

${ }^{30}$ M. Farine, F. W. J. Hekking, P. Schuck, and X. Vinas, Phys. Rev. B 68, 024507 (2003).

${ }^{31}$ H. Heiselberg, Phys. Rev. A 68, 053616 (2003).

${ }^{32}$ A. M. García-García, J. D. Urbina, E. A. Yuzbashyan, K. Richter, and B. L. Altshuler, Phys. Rev. B 83, 014510 (2011).

${ }^{33}$ N. N. Bogoliubov, Uspekhi Fiz. Nauk 67, 549 (1959) [Sov. Phys. Usp. 67, 236 (1959)]

${ }^{34}$ P. G. de Gennes, Superconductivity of Metals and Alloys (W. A. Benjamin, New York, 1966).
${ }^{35}$ A. V. Swidzinsky, Spatially Inhomogeneous Problems in the Theory of Superconductivity (Nauka, Moscow, 1982).

${ }^{36}$ P. W. Anderson, J. Phys. Chem. Solids 11, 26 (1959).

${ }^{37}$ J. B. Ketterson and S. N. Song, Superconductivity (Cambridge University, Cambridge, 1999).

${ }^{38}$ A. L. Fetter and J. D. Walecka, Quantum Theory of Many-Particle Systems (Dover, New York, 2003).

${ }^{39}$ N. W. Ashcroft and N. D. Mermin, Solid State Physics (Saunders, Philadelphia, 1976).

${ }^{40}$ Y. Guo, Y. F. Zhang, X. Y. Bao, T. Z. Tang, L. X. Zhang, W. G. Zhu, E. G. Wang, Q. Niu, Z. Q. Qiu, J. F. Jia, Z. X. Zhao, and Q. K. Xue, Science 306, 1915 (2004).

${ }^{41}$ D. Eom, S. Qin, M. Y. Chou, and C. K. Shih, Phys. Rev. Lett. 96, 027005 (2006); S. Y. Qin, J. Kim, Q. Niu, and C. K. Shih, Science 324, 1314 (2009).

${ }^{42}$ W. Satula, J. Dobaczewski, and W. Nazarewicz, Phys. Rev. Lett. 81, 3599 (1998).

${ }^{43}$ H. Heiselberg and B. Mottelson, Phys. Rev. Lett. 88, 190401 (2002).

${ }^{44}$ G. M. Bruun and H. Heiselberg, Phys. Rev. A 65, 053407 (2002).

${ }^{45}$ J.-P. Martikainen and P. Törma, Phys. Rev. Lett. 95, 170407 (2005).

${ }^{46}$ P. G. de Gennes and D. Saint-James, Phys. Lett. 4, 151 (1963).

${ }^{47}$ C. Caroli, P. G. de Gennes, and J. Matricon, Phys. Lett. 9, 307 (1964).

${ }^{48}$ A. F. Andreev, Zh. Eksp. Teor. Fiz. 49, 655 (1965) [Sov. Phys. JETP 22, 455 (1966)]

${ }^{49}$ A. A. Shanenko, M. D. Croitoru, and F. M. Peeters, Phys. Rev. B 78, 054505 (2008) 